\title{
Thrombosis in VEXAS syndrome
}

\author{
Thet Mon Oo ${ }^{1,2,3}$. Jie Tian Jeanette Koay ${ }^{4}$. Siew Fen Lee ${ }^{1}$. Shang Ming Samuel Lee ${ }^{2,3,5} \cdot$ Xin Rong Lim $^{2,3,5}$. \\ Bingwen Eugene $\operatorname{Fan}^{2,3,4,6}$
}

Accepted: 5 November 2021 / Published online: 24 November 2021

(c) The Author(s), under exclusive licence to Springer Science+Business Media, LLC, part of Springer Nature 2021

\begin{abstract}
VEXAS syndrome, an autoinflammatory syndrome due to a Ubiquitin Like Modifier Activating Enzyme 1 (UBA1) somatic mutation, has a high thrombotic burden. We report a case of a 69-year-old male that was diagnosed with VEXAS syndrome who developed venous thromboembolism (VTE). Review of literature of existing VEXAS syndrome cases showed a high thrombotic burden, with the reported incidence of VTE (36.4\%) being markedly higher than arterial thrombosis (1.6\%), with deep vein thrombosis being more common than pulmonary embolism. Somatic mutation in the UBA1 gene results in decreased ubiquitylation which is a key driver in the development of thrombosis in VEXAS syndrome, due to chronic inflammation and cytokine release from abnormal crosstalk between the intrinsic effector mechanism of innate immune cells, platelets and endothelium resulting in dysregulated haemostasis and endothelial dysfunction. Targeting endothelial dysfunction and reducing inflammatory milieu causing hypercoagulability with immunosuppressants and immunomodulatory agents, together with anticoagulation may be the strategy to prevent recurrent thrombotic events.
\end{abstract}

Keywords Thrombosis · Inflammation · Venous Thromboembolism $\cdot$ Genetics $\cdot$ Mutation $\cdot$ VEXAS Syndrome

\section{Highlights}

- VEXAS syndrome has a high thrombotic burden with a reported incidence of venous thromboembolism $(36.1 \%)$ that is markedly higher than arterial thrombosis $(1.6 \%)$

- X-linked somatic mutation in the UBA1 gene results in decreased ubiquitylation which is a key driver in the development of thrombosis in VEXAS syndrome.

- Chronic cytokine release and inflammation from abnormal crosstalk between the intrinsic effector mechanism

Bingwen Eugene Fan

Bingwen_Eugene_Fan@ttsh.com.sg

1 Department of General Medicine, Tan Tock Seng Hospital, Singapore, Singapore

2 Lee Kong Chian School of Medicine, Singapore, Singapore

3 Yong Loo Lin School of Medicine, Singapore, Singapore

4 Department of Haematology, Tan Tock Seng Hospital, 11 Jln Tan Tock Seng, Singapore 308433, Singapore

5 Department of Rheumatology, Allergy and Immunology, Tan Tock Seng Hospital, Singapore, Singapore

6 Department of Laboratory Medicine, Khoo Teck Puat Hospital, Singapore, Singapore of innate immune cells, platelets and endothelium results in dysregulated haemostasis and endothelial dysfunction.

- Further studies are required to uncover the exact mechanisms of thrombogenesis and to evaluate anticoagulation strategies in patients with VEXAS syndrome.

\section{Introduction}

VEXAS (Vacuoles, E1 enzyme, X-linked, autoinflammatory, somatic) syndrome [1] is described as a late-onset autoinflammatory syndrome exhibiting a spectrum of systemic inflammatory manifestations as well as significant haematologic abnormalities such as macrocytic anaemia, marrow dysplasia, vacuolisation in myeloid cells [2] and thrombosis. Several published case series [1, 3-10] have observed a high prevalence of venous thromboembolism (VTE) in patients with VEXAS syndrome of between $10 \%$ and $56 \%$, with a significant mortality rate $(27 \%-50 \%)$. We report a case of VEXAS syndrome complicated by unprovoked venous thromboembolism, followed by a rapid review of available literature. Our article focuses on the incidence, possible pathogenesis (Fig. 1) and clinical significance of 


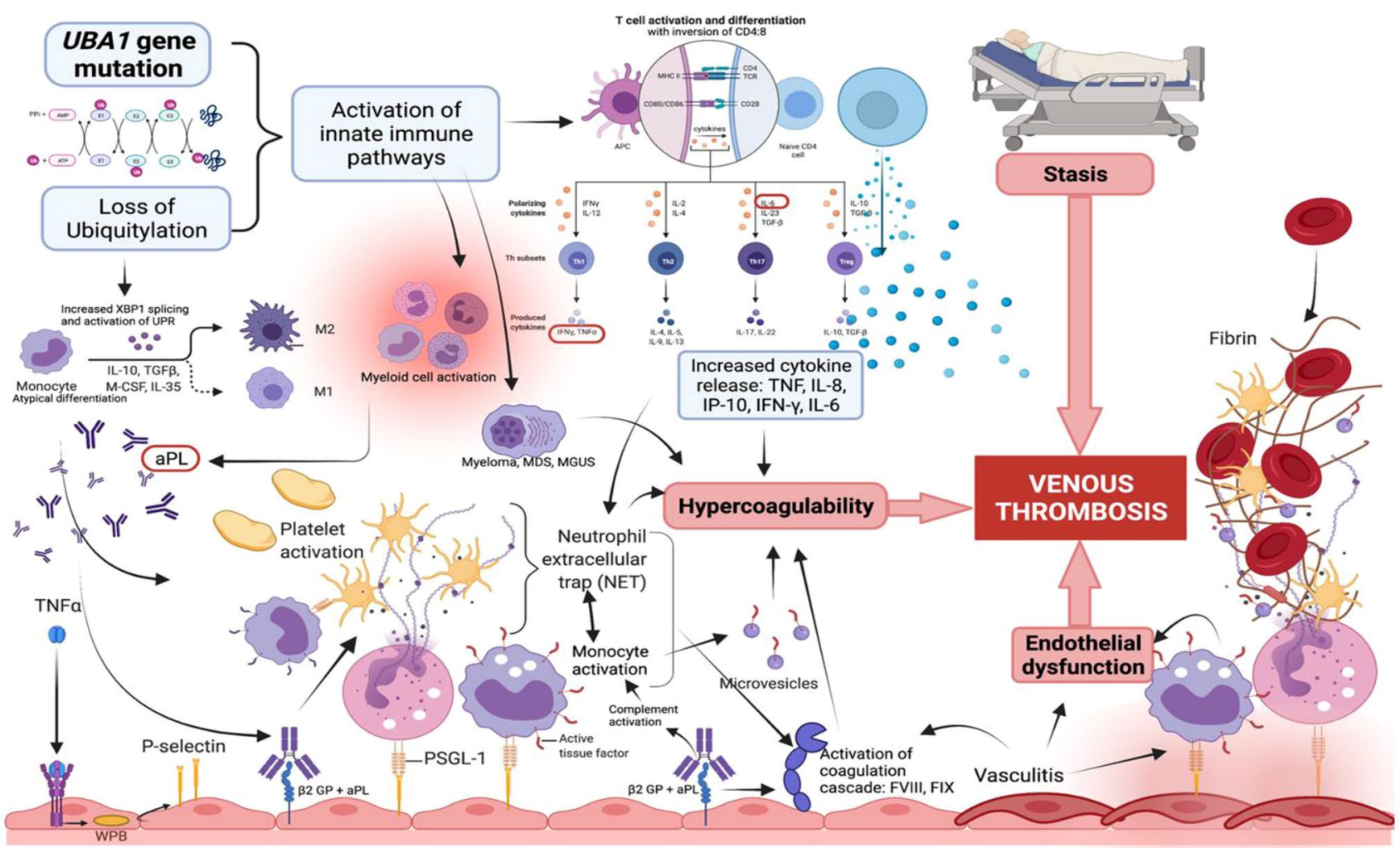

Fig. 1 Virchow's triad showing key molecular and cellular mechanisms of thrombosis in VEXAS syndrome. Somatic mutation of the UBA1 gene on the $\mathrm{X}$ chromosome causes decreased ubiquitylation leading to the activation of innate immune pathways. Subsequently, acquired activation of $\mathrm{B}$ and $\mathrm{T}$ cells gives rise to excess cytokine production, enhanced neutrophil cellular trap formation, uncontrolled monocyte activation, platelet aggregation, and ultimately thrombus formation due to hypercoagulability. Antiphospholipid antibodies contribute towards the activation of platelets and monocytes through complement activation, followed by activation of the coagulation cas-

VTE associated with VEXAS syndrome, which have been minimally discussed in prior publications.

\section{Case report}

A 69-year-old Chinese man with a past medical history of hypothyroidism, hyperlipidaemia, fatty liver, and stage $3 \mathrm{~A}$ chronic kidney disease presented in July 2019 with prolonged fever, urticarial eruption, painful cervical lymphadenitis, migratory arthralgia, myalgia, macrocytic anaemia, and raised C-reactive protein (CRP) and erythrocyte sedimentation rate (ESR). He was treated with methotrexate $12.5 \mathrm{mg} /$ week and a tapering course of prednisolone from $0.5 \mathrm{mg} / \mathrm{kg} /$ day. He later developed relapsing polychondritis and continued to have recurrent flares of painful lymphadenopathy and urticarial rash. cade. Accompanying haematologic malignancies such as myeloma and myelodysplastic syndrome will heighten the hypercoagulability favouring the thrombosis in VEXAS syndrome. Medium-sized vasculitis due to multisystem autoinflammation also leads to further endothelial dysfunction, resulting in a higher risk of thrombosis. $U P R$ unfolded protein response, TNF tumour necrosis factor alpha, $I L-8$ interleukin 8, IL-6 interleukin 6, IFN- $\gamma$ interferon- $\gamma, I P-10$ interferon-inducible protein $10, W P B$ Weibel-Palade bodies, $a P L$ antiphospholipid antibodies, ${ }^{\circledR} 2-G P$ beta2 glycoprotein, $P S G L-1$ P-selectin glycoprotein ligand-1

During an admission in Sept 2020, he presented with acute right lower limb swelling. Ultrasound Doppler scan showed extensive deep vein thrombosis (DVT) extending from the right common femoral vein to the popliteal vein. $\mathrm{He}$ was found to have bilateral pulmonary embolism (PE) on CT thorax (Fig. 2A) and abdomen scans which were performed to exclude cancer-associated thrombosis. His blood investigations showed worsening macrocytic anaemia, mild thrombocytopenia, and persistently raised CRP and ESR. Antiphospholipid antibodies (lupus anticoagulant, anti-cardiolipin $\mathrm{IgG}$ and $\mathrm{IgM}$, anti-B2 glycoprotein I), anti-thrombin III, homocysteine was normal. Slightly depressed protein C activity at $65 \%$ (reference range $70 \%-150 \%$ ) and protein $\mathrm{S}$ activity at $53 \%$ (reference range $65 \%-130 \%$ ) were likely due to his acute thrombosis.

The combination of prolonged fevers, rash, polychondritis, polyarthritis and cytopenias were highly suggestive of VEXAS syndrome. Based on the clinical suspicion of 

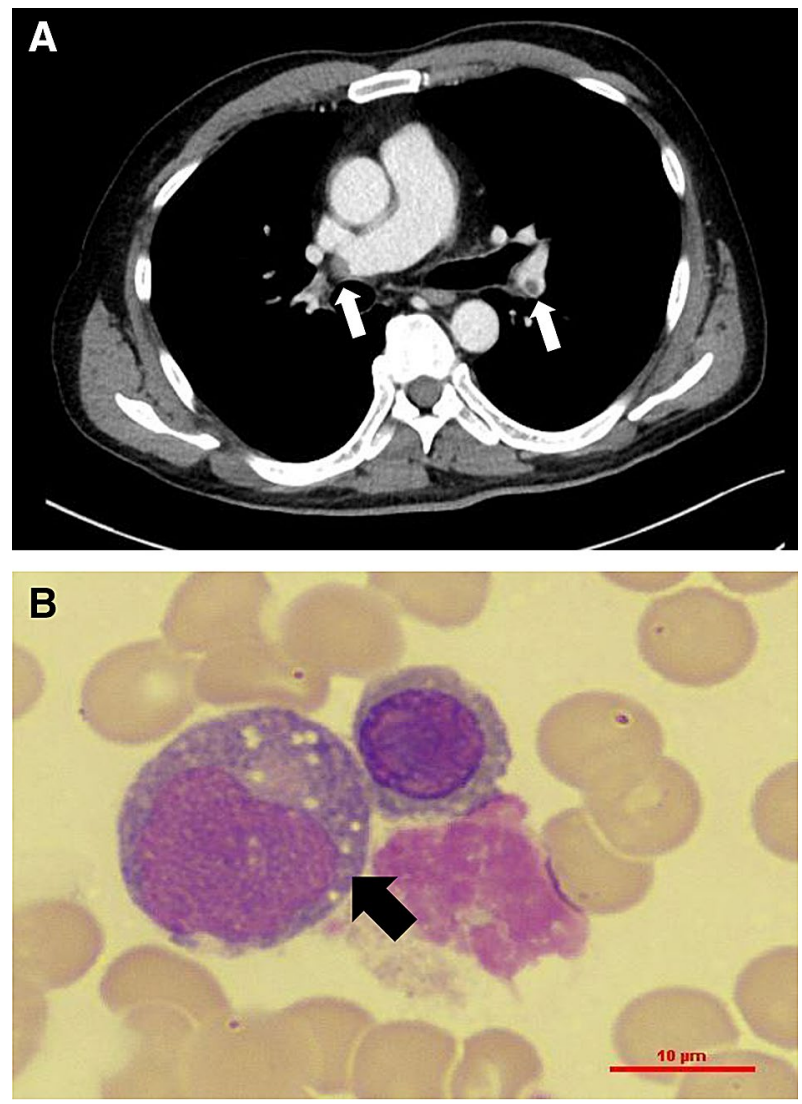

Fig. 2 A: CT thorax showing filling defects (white arrows) in bilateral main pulmonary arteries. B: Bone marrow aspirate with an abnormal myelomonocytic cell (black arrow) with cytoplasmic vacuolation

VEXAS syndrome, a retrospective review of a previous bone marrow examination performed 1 year ago revealed characteristic vacuolation in the myeloid series (Fig. 2B). Sanger sequencing of the peripheral blood showed a somatic mutation of Ubiquitin like Modifier Activating Enzyme 1 (UBA1) variant p.Met41leu (C121A->C), confirming the diagnosis of VEXAS syndrome. Hydroxychloroquine $200 \mathrm{mg} /$ day was added to his treatment regime. For management of his venous thromboembolism, he was initially started on SC enoxaparin $1 \mathrm{mg} / \mathrm{kg} \mathrm{BD}$ and later switched to apixaban $5 \mathrm{mg}$ BD. His right lower limb swelling resolved clinically without any evidence of postthrombotic syndrome. After 6 months of therapeutic anticoagulation, the apixaban dose was reduced to $2.5 \mathrm{mg} \mathrm{BD}$ because of the patient's preference and his concern about bleeding risks. No recurrent episodes of thromboembolic disease were observed in subsequent outpatient reviews.

\section{Discussion}

The first description of VEXAS syndrome [1] reported a significantly high risk of venous thromboembolism of $44 \%$ (11 out of 25 patients). The recently published and largest case series by Georgin-Lavialle et al. [3] described the rate of unprovoked VTE at $35.3 \%$ (41 out of 116 patients). Obiorah et al. [4] reported an even higher VTE rate at $56.25 \%$ (9 out of 16 patients), with $60 \%$ of thrombotic events occurring within the first 2 years of disease onset. 7 patients were observed to have recurrent VTE with a median of 2 events per patient, with 3 patients were noted to have recurrent VTEs despite being on therapeutic anticoagulation. Apart from VTE, there were also arterial thrombotic events reported including transient ischaemic attack (TIA), ischaemic stroke, and myocardial infarction $[4,5]$. A rapid literature review showed that the incidence of VTE $(36.4 \%)$ is markedly higher than arterial thrombosis $(1.6 \%)$. Of the 35 reported VTE cases specifying the location of thrombosis, 33 cases had DVT, while only 8 cases had PE. There were six cases of concurrent DVT and PE (Table 1, supplementary material).

Data on the aetiology and management of VTE was heterogenous, with most studies lacking discussion on these aspects. Obiorah et al. [4] found out of 9 patients with VTE, 2 patients had high factor VIII levels and 1 patient had a high factor IX level, where elevated factor VIII levels are associated with increased risk of venous thrombosis via enhanced thrombin formation. 2 studies evaluated the presence of antiphospholipid antibodies. Beck et al. [1] reported the presence of lupus anticoagulant (LA) in 12 (48\%) of 25 patients with VEXAS syndrome. Obiorah et al. [4] also reported that 7 (44\%) of 16 patients had persistently positive LA and $5(55.5 \%)$ out of 9 patients with VTE had positive LA. One patient also had weakly positive IgM anticardiolipin antibodies. Other tests for thrombophilia included paroxysmal nocturnal haemoglobinuria, antithrombin III activity, and protein $\mathrm{C}$ or $\mathrm{S}$ activity were unremarkable [2]. Although Beck et al reported that UBA1 gene mutations were identified only in males, where males have a higher risk of first and recurrent venous thrombosis than women, 3 studies have reported the development of VEXAS syndrome in 8 females [3, 10, 11]. Moreover, advanced age is an independent risk factor for thrombosis. The median age at onset for VEXAS syndrome is 69.5 years, suggesting predominantly late-onset disease. However, VEXAS can present as early as 45 years of age [1, 4, 5, 9]. Lastly, the association of haematological malignancies with VEXAS syndrome such as myelodysplastic syndrome and multiple myeloma compounds the risk of venous thromboembolism. Table 1 (supplementary material) summarizes 31 reports of 250 patients with VEXAS syndrome. 


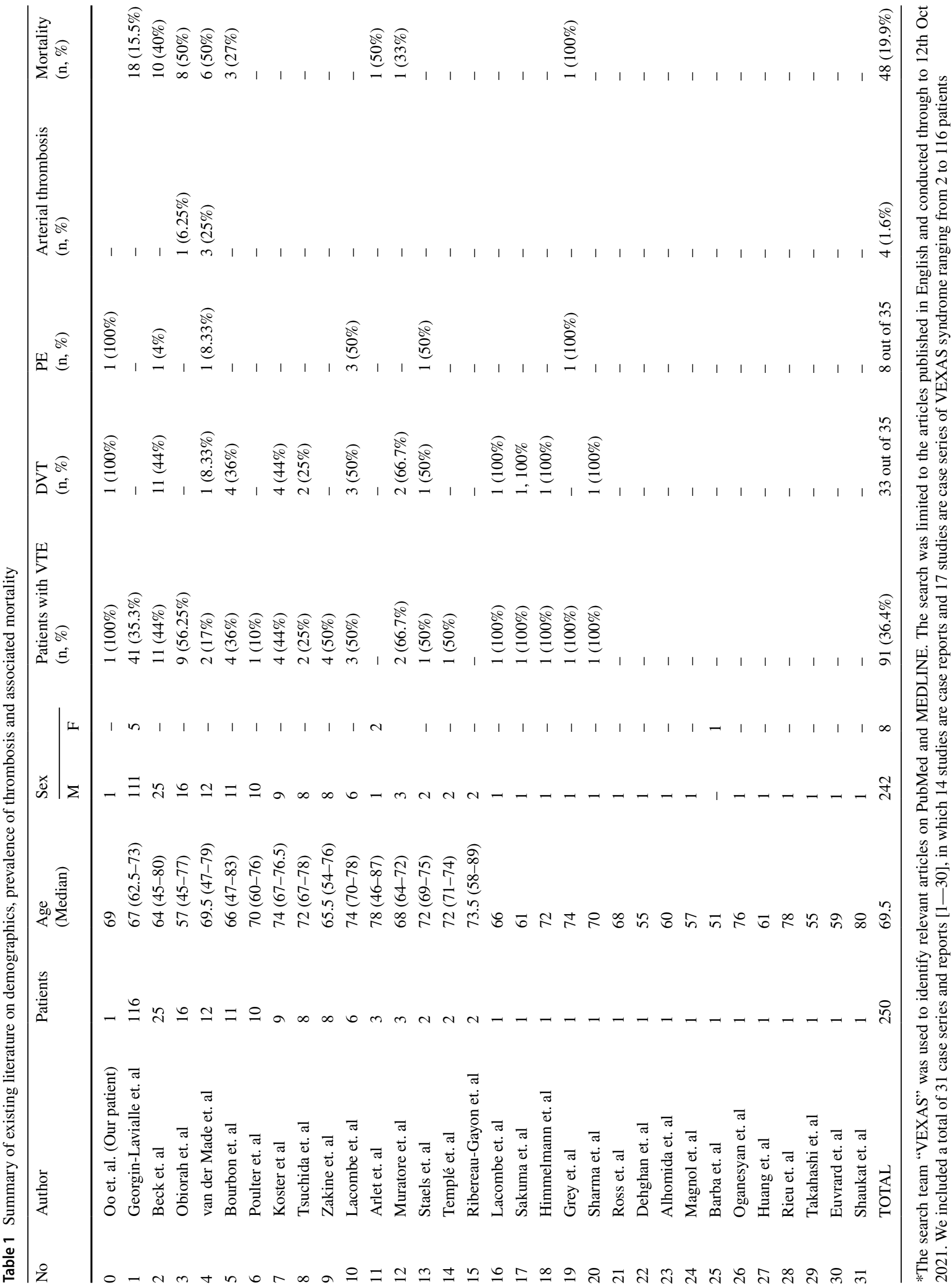


We postulate that the pathogenesis of venous thromboembolism originates from the somatic mutations of methionine-41 (p.Met41) in the UBA1 gene, affecting the major E1 enzyme that initiates ubiquitin conjugation of cellular proteins meant for degradation via proteasomes [1]. Decreased ubiquitination causes accumulation of intra-cellular vacuoles and results in abnormal haematopoietic myeloid precursors, aberrant neutrophils and monocytes, which activate innate immune pathways via cytokine release, causing severe inflammation. An increased percentage of spliced XBP1 in monocytes and activated unfolded protein response (UPR) were also observed, causing monocyte atypical differentiation with loss of non-classical (CD14 dimCD16+) and intermediate monocyte populations $(\mathrm{CD} 14+\mathrm{CD} 16+)[1]$. A sustained overactivation of UPR will result in cellular stress, increased inflammatory response, and eventual apoptosis [12]. Immunoprofiling of $\mathrm{T}$ cells revealed inversion of CD4:CD8 ratio, consistent with an acquired T cell activation consistent with inflammation [1, 4]. Elevated levels of multiple cytokines such as tumour necrosis factor-alpha (TNF- $\alpha$ ), interleukin-8 (IL-8), interleukin-6 (IL-6), interferon-inducible protein 10 (IP-10) and interferon- $\gamma$ (IFN- $\gamma$ ) have been described $[1,13]$. A prothrombotic milieu arises from this cytokine-mediated interaction between endothelial cells, leukocytes, and platelets. Higher levels of pro-inflammatory cytokines upregulate tissue factor (TF) expression by monocytes, macrophages, and endothelial cells [14]. Both IL-6 and IFN- $\gamma$ increase platelet production and activation, giving rise to endothelial activation with release of P-selectin when TNF- $\alpha$ induces the exocytosis of Weibel Palade bodies (WPB) from endothelial cells [15]. Subsequently neutrophils and monocytes bind $\mathrm{P}$-selectin through P-selectin glycoprotein ligand-1 (PSGL-1), triggering recruitment and activation of neutrophils and monocytes [16]. Activated cells express tissue factor and release highly procoagulant microvesicles [17]. Both neutrophils and platelets can activate each other by the classic cytokine-mediated pathways as well as binding of platelets to TREM-1 receptor on neutrophils [18]. In addition, functional studies of aberrant neutrophils [1] showed enhanced spontaneous neutrophil extracellular trap (NET) formation which can lead to endotheliitis, and platelet activation and aggregation. Evidence of cutaneous, pulmonary and systemic vasculitis $[1,4,5]$ as well as ANCA-associated vasculitis [19] reflects multisystem autoinflammation. The presence of pathological antiphospholipid antibodies could enhance thrombogenesis by upregulation of tissue factor production and complement activation, leading to the activation of platelets and monocytes as well as promotion of the coagulation cascade [20]. In summary, decreased ubiquitylation is the key driver in thrombogenesis in VEXAS syndrome, with chronic inflammation from abnormal crosstalk between the intrinsic effector mechanism of innate immune cells, platelets and endothelium resulting in dysregulated haemostasis and endothelial dysfunction. Figure 1 features the key molecular and cellular mechanisms discussed that combine to form Virchow's triad.

Anticoagulation in VEXAS syndrome has not been well described. Apixaban has been used successfully in our patient with no recurrence of VTE. However, current guidelines on the treatment of antiphospholipid syndrome recommend lifelong anticoagulation with vitamin $\mathrm{K}$ antagonists instead of DOACs. Therefore, evaluation for antiphospholipid antibodies in all patients with VEXAS syndrome may be an important consideration before DOAC use. While anticoagulation targets the hypercoagulable state in Virchow's triad, endothelial damage remains a key contributing factor to thrombosis. Currently, a variety of treatments with glucocorticoids, immunosuppressive drugs and immune-modulating therapies have been used to treat VEXAS syndrome but no treatment paradigms have been established. A strategy targeting endothelial dysfunction and reducing inflammatory milieu causing hypercoagulability with these agents with anticoagulation may prevent recurrent thrombotic events.

\section{Conclusion}

Given that chronic autoinflammation and increased cytokine release are the key pathophysiological processes in driving hypercoagulability and endothelial dysfunction, early recognition of VEXAS syndrome and management with immunosuppressants and immune-modulating therapies may help mitigate the risk of thromboembolic disease. Further studies are required to uncover the exact mechanisms of thrombogenesis and evaluate anticoagulation strategies in patients with VEXAS syndrome.

Supplementary Information The online version contains supplementary material available at https://doi.org/10.1007/s11239-021-02608-y.

Acknowledgements The authors greatly appreciate the efforts of our fellow healthcare workers during this COVID-19 pandemic.

Authors' contribution BEF conceived the study. All authors contributed substantially to the acquisition, analysis and interpretation of data, critical revision of manuscript for important intellectual content.

Funding No funding was required for this study.

Data availability The data that support the findings of this study are available from the corresponding author upon reasonable request.

\section{Declarations}

Conflict of interest The authors declare that they have no conflict of interest.

Ethical approval Written consent was obtained from the patient. 


\section{References}

1. Beck DB, Ferrada MA, Sikora KA et al (2020) Somatic mutations in $U B A 1$ and severe adult-onset autoinflammatory disease. N Engl J Med 383(27):2628-2638. https://doi.org/10.1056/NEJMoa2026 8342

2. Fan BE, Cao L, Gallardo CA et al (2021) Myeloid and lymphoid vacuolation in VEXAS syndrome. Am J Hematol 96(8):10561057. https://doi.org/10.1002/ajh.26098

3. Georgin-Lavialle S, Terrier B, Guedon AF et al (2021) Further characterization of clinical and laboratory features occurring in VEXAS syndrome in a large-scale analysis of multicenter caseseries of 116 French patients. Br J Dermatol. https://doi.org/10. 1111/bjd.20805

4. Obiorah IE, Patel BA, Groarke EM et al (2021) Benign and malignant hematologic manifestations in patients with VEXAS syndrome due to somatic mutations in UBA1. Blood Adv 5(16):3203-3215. https://doi.org/10.1182/bloodadvances.20210 04976

5. van der Made CI, Potjewijd J, Hoogstins A et al (2021) Adultonset autoinflammation caused by somatic mutations in UBA1: a Dutch case series of patients with VEXAS. J Allergy Clin Immunol. https://doi.org/10.1016/j.jaci.2021.05.014

6. Bourbon E, Heiblig M, Gerfaud Valentin M et al (2021) Therapeutic options in VEXAS syndrome: insights from a retrospective series. Blood 137(26):3682-3684. https://doi.org/10.1182/blood. 2020010177

7. Poulter JA, Collins JC, Cargo C et al (2021) Novel somatic mutations in UBA1 as a cause of VEXAS syndrome. Blood 137(26):3676-3681. https://doi.org/10.1182/blood.2020010286

8. Tsuchida N, Kunishita Y, Uchiyama Y et al (2021) Pathogenic $U B A 1$ variants associated with VEXAS syndrome in Japanese patients with relapsing polychondritis. Ann Rheum Dis. https:// doi.org/10.1136/annrheumdis-2021-220089

9. Lacombe V, Prevost M, Bouvier A et al (2021) Vacuoles in neutrophil precursors in VEXAS syndrome: diagnostic performances and threshold. Br J Haematol. https://doi.org/10.1111/bjh.17679

10. Arlet JB, Terrier B, Kosmider O (2021) Mutant UBA1 and severe adult-onset autoinflammatory disease. N Engl J Med 384(22):2163. https://doi.org/10.1056/NEJMc2102124
11. Barba T, Jamilloux Y, Durel CA et al (2021) VEXAS syndrome in a woman. Rheumatology (Oxford). https://doi.org/10.1093/rheum atology/keab392

12. Ivanova EA, Orekhov AN (2016) The role of endoplasmic reticulum stress and unfolded protein response in atherosclerosis. Int $\mathbf{J}$ Mol Sci. 17(2):193. https://doi.org/10.3390/ijms17020193

13. Grey A, Cheong PL, Lee FJ et al (2021) A case of VEXAS syndrome complicated by hemophagocytic lymphohistiocytosis. J Clin Immunol 41(7):1648-1651. https://doi.org/10.1007/ s10875-021-01070-y

14. Najem MY, Couturaud F, Lemarié CA (2020) Cytokine and chemokine regulation of venous thromboembolism. J Thromb Haemost 18(5):1009-1019

15. McCormack JJ, Lopes da Silva M, Ferraro F, Patella F, Cutler DF (2017) Weibel-Palade bodies at a glance. J Cell Sci 130(21):36113617. https://doi.org/10.1242/jcs.208033

16. Engelmann B, Massberg S (2013) Thrombosis as an intravascular effector of innate immunity. Nat Rev Immunol 13(1):34-45. https://doi.org/10.1038/nri3345

17. Borissoff JI, ten Cate H (2011) From neutrophil extracellular traps release to thrombosis: an overshooting host-defense mechanism? J Thromb Haemost 9(9):1791-1794. https://doi.org/10.1111/j.15387836.2011.04425.x

18. Rautou PE, Mackman N (2013) Microvesicles as risk markers for venous thrombosis. Expert Rev Hematol 6(1):91-101. https://doi. org/10.1586/ehm.12.74

19. Muratore F, Marvisi C, Castrignanò P et al (2021) VEXAS syndrome: a case series from a single-center cohort of Italian patients with vasculitis. Arthritis Rheumatol. https://doi.org/10.1002/art. 41992

20. Meroni PL, Borghi MO, Raschi E, Tedesco F (2011) Pathogenesis of antiphospholipid syndrome: understanding the antibodies. Nat Rev Rheumatol 7(6):330-339

Publisher's Note Springer Nature remains neutral with regard to jurisdictional claims in published maps and institutional affiliations. 